\title{
Real Time Optical Patient Surface Motion Monitoring During Radiotherapy
}

\author{
Gareth PRICE*, James PARKHURST, Phil SHARROCK, Chris MOORE \\ North Western Medical Physics, The Christie NHS Foundation Trust, Manchester, UK
}

\begin{abstract}
The increasing use of aggressive radiotherapy treatment regimes means that patient monitoring during treatment delivery is assuming greater importance in image guided radiotherapy. Optical metrology to measure and correct bulk positional offsets has previously been demonstrated in this respect, but systems have not had the capability to offer high spatial and temporal resolution dynamic monitoring of wide field skin surfaces. We describe the implementation of a genuinely real-time optical skin surface measurement system, based on Fourier profilometry, and its clinical application in a radiotherapy treatment room. The system is capable of processing (image capture through to display) $\sim 23$ frames per second at 512x512 resolution. Three camera-projector pairs may be operated synchronously to provide a 'wrap-around' surface, overcoming the line of sight limitations of the structured light projection approach. In addition to describing our implementation of the surface calculation algorithm, we additionally describe an application that we are currently evaluating in a volunteer study: the use of visual feedback of live body surfaces to reduce patient motion.
\end{abstract}

Keywords: Fourier profilometry, image guided radiotherapy, patient motion, medical imaging

\section{Introduction}

The aim in radiotherapy is to deliver a therapeutic radiation dose to a tumour whilst avoiding any nearby healthy tissues. Typically a patient's treatment is numerically simulated on an X-ray CT scan. The body surface, tumour, and other healthy anatomy is delineated in three dimensions and the X-ray beam geometry configured to maximize the dose to the cancer target whilst sparing the skin and other critical structures. Patient movement during irradiation is known, however, to compromise treatment. Should the tumour not receive its prescribed dosage there is the risk of local recurrence, i.e. failure of the therapy, whilst damaging or destroying healthy tissues can lead to very serious side effects. The use of imaging techniques to measure and account for any motion that could have a deleterious effect on outcome, has, therefore, become an integral part of modern image guided radiotherapy (IGRT).

The majority of the imaging modalities used in this way, e.g. 4D CT, dynamic MRI and cone beam CT, are presently limited to being deployed immediately prior to or following treatment, not during the actual radiation delivery. In this context 3D optical measurement of patient skin surfaces becomes attractive. Although self-evidently unable to image internal anatomy, monitoring for surface motion, for example checking for movement outside of acceptable bounds, is vital, especially so as increasingly aggressive treatments, such as hypo-fractionation and proton beam therapy, become available. With scanning beam proton therapy in particular, changes in skin surface can have an appreciable effect on the dose received by the tumor and the surrounding healthy tissue.

Various non-contact optical measurement technologies have the ability to measure changes in skin topology. However, these techniques are often computationally demanding and, as yet, have only delivered low frame rates in the radiotherapy environment. A typical example would be the use of stereo-photogrammetric methods which, as far as the authors are aware, have reported maximum wide field performance of only $\sim 2 \mathrm{~Hz}$ [1]. At such sampling frequencies many anatomical movements will not be visible. In the context of visualization applications, frame rates greater than $20 \mathrm{~Hz}$ are commonly considered to be 'real-time'. Henceforth we use this definition of real-time for surface topology measurement.

In this paper we present the development of a genuinely real-time structured light optical surface measurement approach suitable for use in image guided radiotherapy [2, 3]. Additionally we describe the clinical deployment of the system to provide real-time feedback of patient position and pose in support of improved immobilization during irradiation [4]. The operating principle of our system is Fourier profilometry, in which all of the information required to calculate surface topology can be extracted from a single video frame. Accordingly, it can be used for wide field, 'real-time' measurement and control providing that the combined projection, video-capture, processing and display times become fast enough.

* gareth.price@physics.cr.man.ac.uk; +44 (0)161 4463258 
Our implementation directly measures the patient's skin surface at 512 by 512 pixel resolution and is capable of dynamic measurement at $23 \mathrm{~Hz}$. The accuracy of the system is, to a large degree, dictated by the system geometry, which, in the radiotherapy treatment room, is highly non-optimal. Nevertheless, the accuracy of the measured points, within a $40 \mathrm{~cm}^{3}$ calibration volume about the linear accelerator treatment isocentre, agree with equivalent cone beam CT images to within $2 \mathrm{~mm}$ (often approaching $1 \mathrm{~mm}$ or better). The main focus of this paper will be the system development required to achieve this performance, although as stated above, we additionally briefly introduce one of its current clinical applications.

\section{Materials and methods}

In structured light topology measurement systems in general, the height of an object is found through the analysis of the modulation of a fringe pattern projected onto it. In Fourier profilometry in particular, the changes in spatial phase of the pattern are recovered through filtering in Fourier space using the well known methodology of Takeda et al. [5]. One can monitor for motion simply by looking for changes in the spatial phase, but should accurate quantitative measurements be required, the relationship between the phase and world coordinates needs to be established. We calibrate relative to a flat reference plane, and use spots within the projected pattern to uniquely identify pattern fringes. We therefore calibrate against the absolute measured change in pattern spatial phase to give the absolute world coordinates relative to a coordinate frame of reference.

In our application, severe geometrical constraints are imposed on the location of the system projector and camera by the large rotating gantry of the linear accelerator, and by the clinical procedures in place. The practical outcome of this is that the cameras are setup some distance from, and at an oblique angle to, the patient. This causes shadowing in regions that aren't visible to the camera and limits the area of the patient's body that can be measured using a single sensor system. To overcome this problem we combine three camera-projector pairs separated by narrowband colour filters. Figure 1 shows the geometry of the system relative to the linear accelerator gantry and treatment couch. The central green light sensor is setup such that it is always possible to measure the patient; the left (red light) and right (blue light) sensors are setup to provide the wrap-around capability; however they can become partially occluded during gantry rotation. Narrowband colour channel separation of the sensor cameras and projectors comes at the cost of a decreased signal-to-noise ratio in each channel.

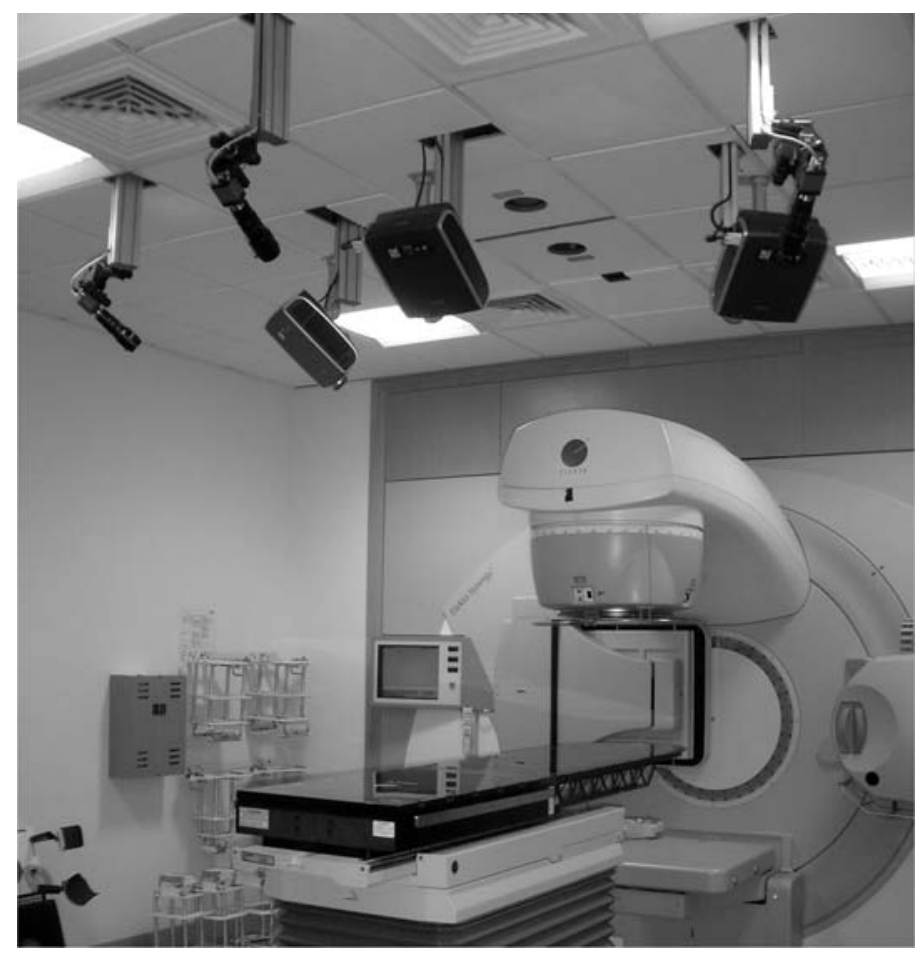

Fig. 1. The three colour channel separated camera-projector pairs of our Fourier profilometry optical surface measurement system in a radiotherapy treatment room at The Christie. The rotating gantry and imaging panels constrain the geometry of the system to the highly non-optimal arrangement pictured. The camera and projector axes have incident angles of approximately $40^{\circ}$. 
Each sensor 'head' consists of a Proslica (Prosilica Inc., Burnaby, Canada) GE1380 CCD camera equipped with a Navitar (Navitar Inc., Rochester, USA) Zoom 7000 lens and a Canon (Canon UK Ltd., Woodhatch, UK) SX60 data projector. An Intel Core 2 Quad $3 \mathrm{GHz}$ PC with two Nvidia (Nvidia Corporate, Santa Clara, CA, USA) GTX480 graphics cards is used to control the projectors, with a single high performance computer used for image capture and processing from all three head units. The processing computer has two Intel Xeon X5680 processors giving a machine total of 12 CPU cores and 24 separate hyper-threads of execution.

The system software is written in the $C$ programming language and utilizes the Intel (Intel Corporation, Santa Clara, USA) optimized Intel Integrated Performance Primitives (IPP) library. We parallelize the major processing blocks using the standard Microsoft Windows threading libraries. We use three major threads: one to perform camera image acquisition, the second to carry out the profilometry calculations, and the third for surface display via OpenGL. Another separate thread monitors the graphical user interface.

Our implementation of the Fourier profilometry algorithm can be split into the following four processing steps: calculation of the reference spot coordinates used to determine the absolute change in phase of the fringe pattern from a flat reference plane; extraction of the wrapped (i.e. modulo $2 \pi$ ) spatial phase map from the fringe image; calculation of the unwrapped phase distribution; and conversion from the absolute phase values to calibrated real-world coordinates. A description of each of these algorithm stages is given below.

\subsection{Calculation of reference spot coordinates}

Reference spots are used to identify the location of a specific fringe in each fringe image. By tracking how the fringe moves with changes in object topology relative to a flat reference plane, the absolute change in pattern spatial phase is found, enabling quantitative calibration in room coordinates.

Though there are various pitfalls to try and avoid in the accurate detection of spots in images, the general algorithm employed here is very simple, easy to implement and computationally efficient. The spots are isolated from the background by applying the Laplacian of Gaussian operation to the fringe image. Since the spot size is found during calibration of the system, multi-scale operations are not required. The spots are then identified by searching along the track traversed across the camera CCD by each spot as the flat reference plane is raised and lowered through the calibration volume.

The spot tracks are well-defined and by design do not overlap at any point, and thus each can be easily identified from frame to frame. The computation time required for the calculation of the coordinates of the seven reference spots we use (for reasons of redundancy) is $\sim 5 \mathrm{~ms}$.

\subsection{Calculation of wrapped phase map}

We fully describe the calculation of wrapped (modulo $2 \pi$ ) spatial phase elsewhere [6], broadly following the methodology of Takeda and Mutoh [7]. Briefly, the processing can be summarized as follows. The image is first filtered in order to removing the background leaving the fringes as the major component. The Fourier transform of the image is then calculated and the image filtered in the frequency domain using a band-pass filter to isolate the spectral peak corresponding to the frequency of the fringes. When the filtered spectrum is inverse transformed, the wrapped phase map may be calculated as the imaginary part of the natural logarithm of the resulting complex image. This is illustrated in Figure 2.

Using the Fourier transforms and various image processing functions available in the Intel IPP libraries, and applying micro-optimizations to the image processing source code, the computation time required for the calculation of the wrapped phase map from the input fringe image is $\sim 15 \mathrm{~ms}$. 
a)

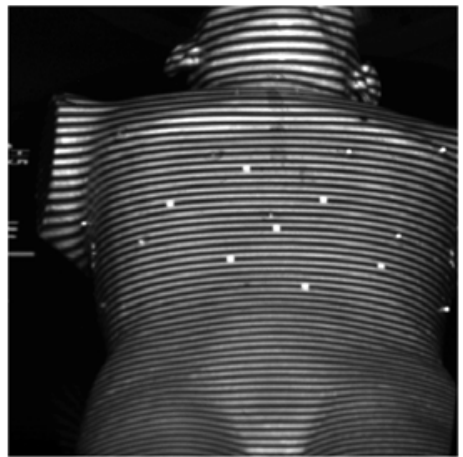

d)

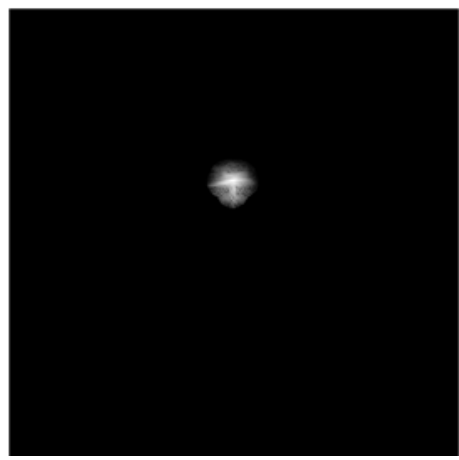

b)

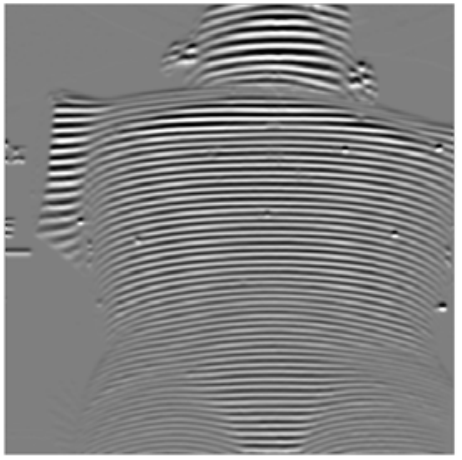

c)

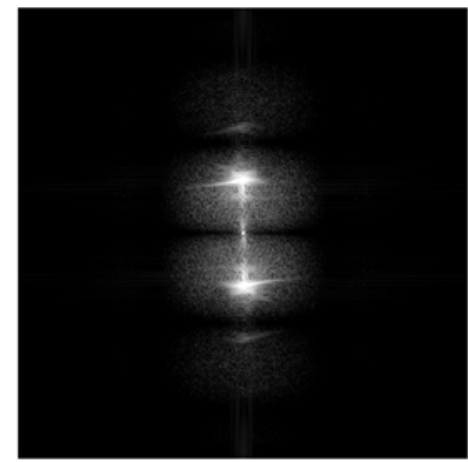

e)

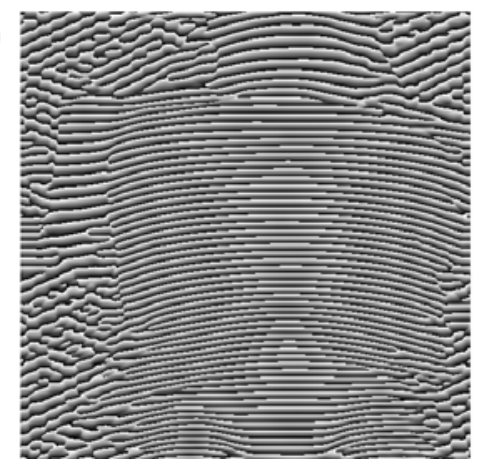

f)

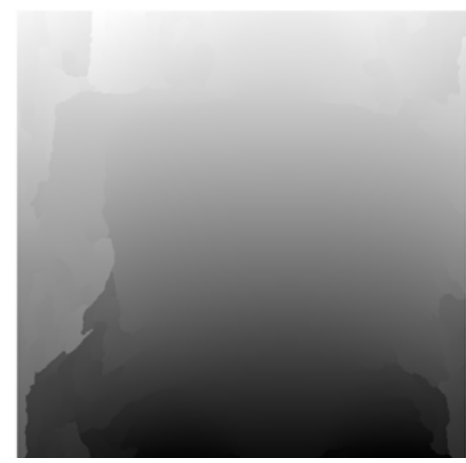

Fig. 2. Extraction of the spatial phase from a modulated sinusoidal fringe pattern. Here the subject is an anthropomorphic phantom. The raw image captured by the camera (a) first has the reference spots and background texture removed (b) prior to Fourier transformation (c). In Fourier space one of the transform's first order maxima is isolated (d). The inverse Fourier transform of the filtered spectra then yields the phase of the fringe pattern (e). The phase is initially modulo $2 \pi$ but can be unwrapped to give the continuous distribution ( $f$ ).

\subsection{Calculation of unwrapped phase map}

The imaginary part of the complex natural logarithm is equivalent to its complex argument. The spatial phase calculated as described above is therefore revealed as the periodic result of the inverse tangent function: the distribution values are wrapped between $\pi$ and $-\pi$. In order to find the true changes in spatial phase across the field of view of the instrument, a phase unwrapping algorithm needs to be employed.

At the time of writing, there are no publicly available software libraries, commercial or otherwise, that implement a selection of general purpose phase unwrapping algorithms. It is therefore necessary to either use an available research implementation, or to implement an algorithm directly from its published description. An investigation, fully described elsewhere [8], was conducted in order to identify those algorithms best suited for use in our application, i.e. robust, high speed and precise operation on human body topology. A great number of phase unwrapping algorithms exist in the literature; however, only those with a readily available implementation were used in the investigation [9-11]. 
a)

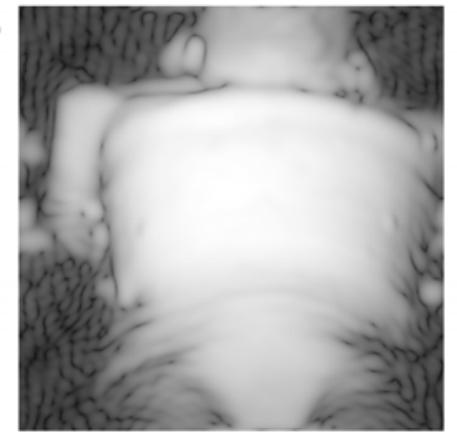

b)

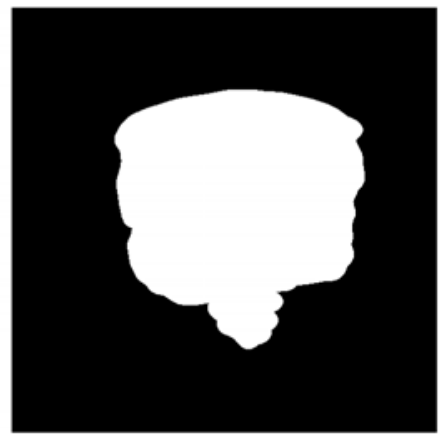

c)

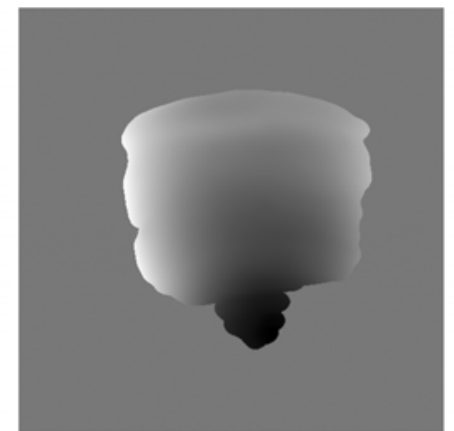

d)

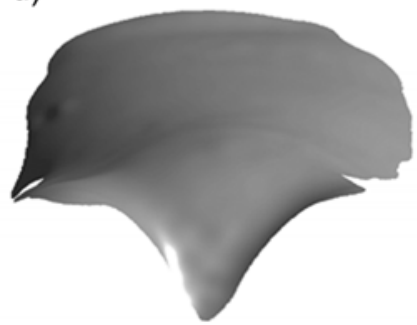

e)

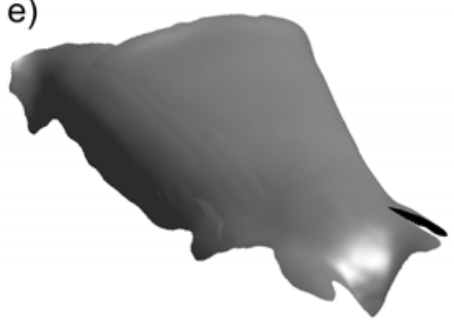

Fig. 3. Dynamic masking of phase distribution and calibration to room coordinates. The absolute inverse Fourier transform (a) of the filtered spectrum shown in Fig. $2 d$ may be thresholded to create a dynamic, per frame mask (b). Applying the mask to the unwrapped phase distribution (Fig. 2f) isolates the surface region of interest (c). The final phase map is calibrated in world coordinates (see [6]) through the application of simple parametric equations whose coefficients are determined a-priori $(d)$. The surfaces from the three separate systems combine to give the full wrap-around object topology (e).

Ideally, an algorithm would combine robust performance with high speed of execution. Inevitably in reality, one must compromise between the two. None of the algorithms we tested met our exacting specifications. Execution times ranged between $13 \mathrm{~ms}$ and $5 \mathrm{~s}$ for the wrapped distributions we used, but the faster performing implementations failed to provide the stable performance clinical use demands. We modified an existing algorithm we concluded had the requisite accuracy - the continuous quality-guided path algorithm described in [10] - to reduce its run time. The resulting algorithm introduces a parameter-free preprocessing step to partially unwrap the phase map before applying the unmodified algorithm to complete the process. There were no observable differences between the resulting unwrapped phase maps and those from the unmodified algorithm.

Using the modified algorithm, a full 512x512 pixel phase map can be unwrapped in $\sim 30 \mathrm{~ms}$. However, following the calculation of the wrapped phase distribution we apply a binary mask which isolates the region of interest, typically $\sim 40 \%$ of the full image (see Figure 3 ). The mask is dynamically generated from the absolute values of the inverse Fourier transform of the filtered spectrum on a per-frame basis. The algorithm is able to unwrap the masked phase map in $\sim 9 \mathrm{~ms}$.

\subsection{Conversion to real world coordinates}

In order to convert the relative phase map into a full 3D surface, three simple equations, whose parameters are found during calibration of the system, are applied to each of the phase values. The height ( $y$ coordinate) at each image point is calculated by application of the non-linear equation resulting from the three dimensional geometric analysis of the system. The $x$ and $z$ world coordinates are then calculated through a hyper-quadratic function of the camera CCD $\mathrm{x}$ and $\mathrm{y}$ pixels, and the object height each pixel maps to. The computation time required for this step, $\sim 1 \mathrm{~ms}$, is negligible compared to that of finding the spots and calculating the unwrapped phase map. Figure $3 \mathrm{~d}$ shows the example unwrapped phase map transformed to world coordinates.

\section{Results}

\subsection{System performance}

The execution times for each stage of our implementation of the Fourier profilometry algorithm are shown in Table I. Based of these measurements alone we have a theoretical frame rate of $33 \mathrm{~Hz}$. The maximum camera capture rate of $25 \mathrm{~Hz}$, however, constrains this speed. Once one has taken this 
maximum into account together with data transfer, and considered thread synchronization and other housekeeping overheads, we achieve a speed of $\sim 23$ frames per second.

This performance is good enough to allow seemless operation and, more importantly, to capture the anatomical motion that our application seeks to monitor. If all three color-separated data channels are used, the system is currently capable of performing synchronized acquisition to display at a rate of $\sim 16 \mathrm{fps}$. The slow down is attributed to the increased thread synchronization burden.

Table 1. Execution times for each algorithm step

\begin{tabular}{|l|c|}
\hline \multicolumn{1}{|c|}{ Processing Step } & Computation Time \\
\hline Spot Coordinates & $5 \mathrm{~ms}$ \\
\hline Wrapped Phase & $15 \mathrm{~ms}$ \\
\hline Unwrapped Phase & $9 \mathrm{~ms}$ \\
\hline Real-world Coordinates & $<1 \mathrm{~ms}$ \\
\hline
\end{tabular}

Comparison of repeat surface measurements of an anthropomorphic phantom (as seen in Figures 2 and 3) with simultaneously captured cone beam CT images shows better than $2 \mathrm{~mm}$ agreement over $90 \%$ of the intersecting regions of interest, and better than $1 \mathrm{~mm}$ over $62 \%$. A typical patient image is shown in Figure 4.
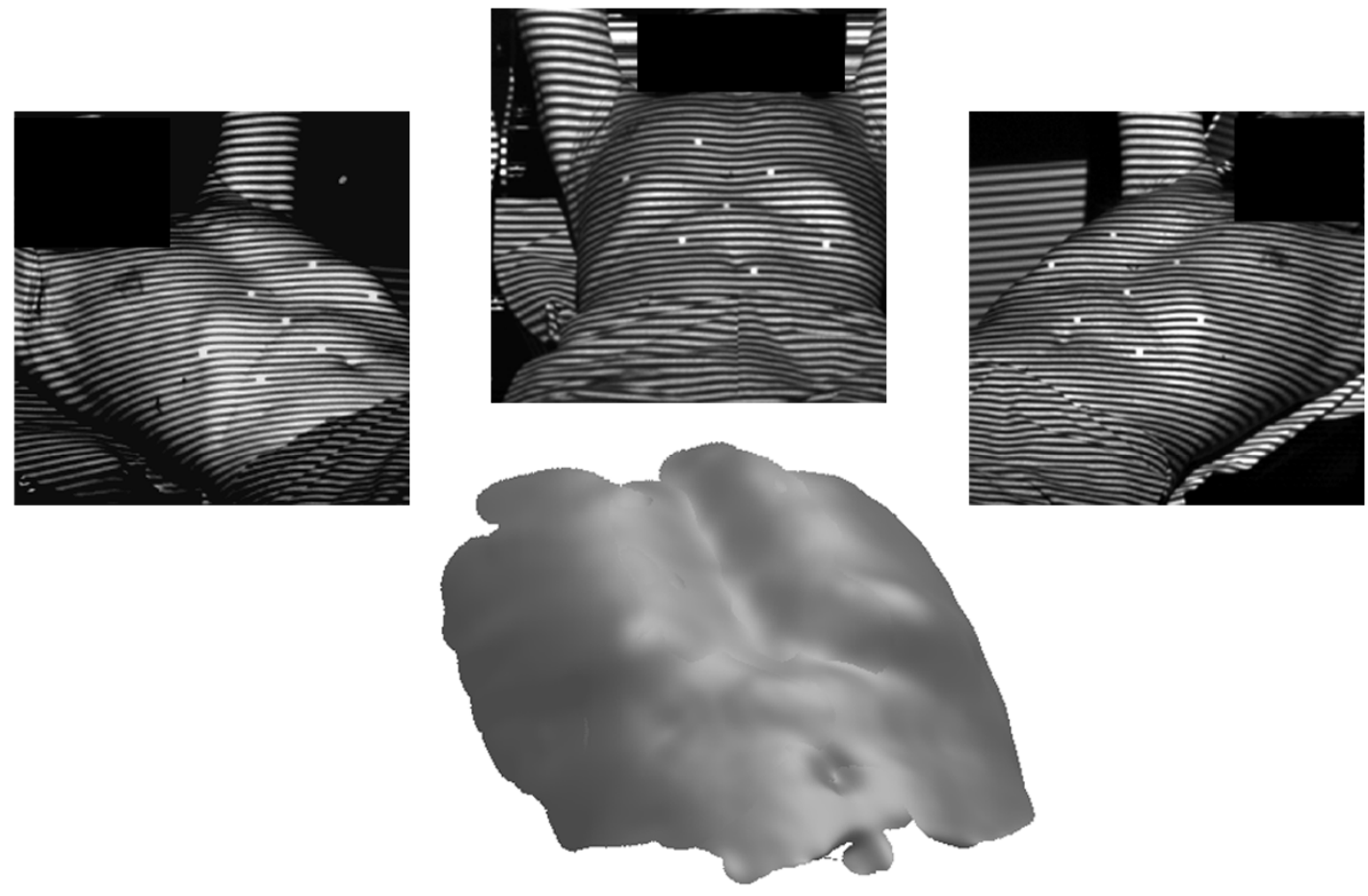

Fig. 4. An illustrative surface, calculated from the three displayed images.

\subsection{Clinical application: visual feedback}

As an illustrative guide to the potential clinical utility of real-time optical surface measurement in radiotherapy, in this section we describe the use of our system in providing visual feedback to the patient. The aim of this approach is to present the patient with information about their position in relation to a reference surface, and thereby enable them to keep within prescribed boundaries. Less motion of course, translates to improved targeting during therapy.

Following consultation with patient representative groups, it emerged that the concept of the 'lamina', the residual surface created by subtracting the live measured surface from the reference surface, was a more intuitive visualization than the actual measured topology when trying to maintain poise within certain bounds. Figure 5 shows a snapshot of a typical surface lamina. As the patient moves, the lamina flexes, moving either side of the centerline representing the reference surface. We additionally colour regions of the surface depending on distance from zero deviation, moving from green for acceptable, through amber to red for unacceptable. 


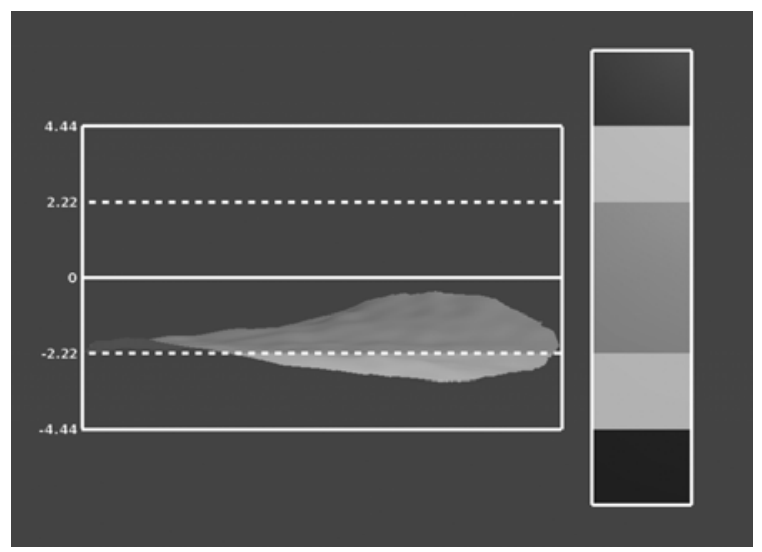

Fig. 5. Visualization of patient motion relative to a reference surface. The lamina represents the residual distance of the live surface from the reference. Threshold bars and colours are used to indicate the range of acceptable motion.

In order to provide an effective visualization that displays the patient's motion smoothly and intuitively, video frame rate performance is a necessity. Lower frame-rates could result in a visualization that appears discontinuous. Speed is especially important when providing visual feedback to patients, since the smoother the visualization appears, the easier it will be for them to interpret their motion.

\section{Conclusion}

We have presented an optical surface measurement system, based on Fourier profilometry, that is suitable for use in the radiotherapy treatment room. The system provides genuinely real-time data at a rate great enough to enable its use, amongst other applications, in providing smooth visual feedback to patients.

It should be noted that our implementation does not represent the ultimate performance of the methodology, merely that of a system using modest acquisition hardware under demanding and highly non-optimal conditions. Bearing this in mind, we believe that a system operating on the principles we have described will make a welcome and timely addition to the medical image guidance toolset.

\section{References}

1. J.L. Peng et. al., "Characterization of a real-time surface image-guided stereotactic positioning system", Med.Phys., 37 (10) p5421-5433 (2010)

2. C. Moore et. al., "Opto-electronic sensing of body surface topology changes during radiotherapy for rectal cancer", Int.J.Rad.Oncol.Biol.Phys, 56 (1) p248-258 (2003)

3. G.J. Price et. al., "An analysis of breast motion using high frequency, dense surface points captured by optical sensor during radiotherapy treatment delivery", Phys.Med.Biol., 54 p6515-6533 (2009)

4. J.M. Parkhurst et. al., "Visualisation Of Dynamic Surface Data For A Patient Display To Reduce Movement During Radiotherapy", in Proceedings of the 7th IASTED Biomedical Engineering (BIOMED-2010) Conference, Innsbruck, 2, p76-82 (2010)

5. M. Takeda et. al., "Fourier-Transform Method of Fringe-Pattern Analysis for Computer-Based Topography and Interferometry", J.Opt.Soc.Am., 72 (1) p156-160 (1982)

6. G.J. Price et. al., "Real time optical measurement of the dynamic body surface for use in guided radiotherapy", Phys.Med.Biol., (submitted July 2011)

7. M. Takeda and K. Mutoh, "Fourier-Transform Profilometry for the Automatic-Measurement of 3-D Object Shapes", App.Opt., 22 (24) p3977-3982 (1983)

8. J.M. Parkhurst et. al., "Selecting a phase unwrapping algorithm for use in a real-time optical body sensor system for use during radiotherapy", App.Opt., (submitted May 2011)

9. D.C. Ghiglia and M.D. Pritt, "Two dimensional phase unwrapping: Theory, algorithms and software", Wiley (1998)

10. M.A. Herraez et. al., "Fast two-dimensional phase-unwrapping algorithm based on sorting by reliability following a noncontinuous path", App.Opt., 41 (35) p7437-7444 (2002)

11. M.A. Herraez et. al., "Robust, fast, and effective two-dimensional automatic phase unwrapping algorithm based on image decomposition", App.Opt., 41 (35) p7445-7455 (2002) 